\title{
Effect of Resistance Training on Physical Disability in Chronic Heart Failure
}

\author{
Patrick Savage ${ }^{1}$, Anthony O. Shaw ${ }^{1}$, Mark S. Miller ${ }^{2}$, Peter VanBuren ${ }^{1,2}$, Martin M. \\ LeWinter ${ }^{1,2}$, Philip A. Ades ${ }^{1}$, and Michael J. Toth ${ }^{1,2}$ \\ ${ }^{1}$ Department of Medicine, University of Vermont, College of Medicine, Burlington, VT 05405 \\ 2Department of Molecular Physiology and Biophysics, University of Vermont, College of Medicine, \\ Burlington, VT 05405
}

\section{Abstract}

Purpose-Patients with chronic heart failure (CHF) report difficulty performing activities of daily living. To our knowledge, however, no study has directly measured performance in activities of daily living in these patients to systematically assess their level of physical disability. Moreover, the contribution of skeletal muscle weakness to physical disability in CHF remains unclear. Thus, we measured performance in activities of daily living in CHF patients and controls, its relationship to aerobic capacity and muscle strength and the effect of resistance exercise training to improve muscle strength and physical disability.

Methods-Patients and controls were assessed for performance in activities of daily living, selfreported physical function, peak aerobic capacity, body composition and muscle strength before and after an 18-wk resistance training program. To remove the confounding effects of several disease-related factors (muscle disuse, hospitalization, acute illness), we recruited controls with similar activity levels as CHF patients and tested patients $>6$ months following any disease exacerbation/hospitalization.

Results-Performance in activities of daily living was $30 \%$ lower $(\mathrm{P}<0.05)$ in CHF patients versus controls and was related to both reduced aerobic capacity $(\mathrm{P}<0.001)$ and muscle strength $(\mathrm{P}<0.01)$. Moreover, resistance training improved $(\mathrm{P}<0.05$ to $<0.001)$ physical function and muscle strength in patients and controls similarly, without altering aerobic capacity.

Conclusion-CHF patients are characterized by marked physical disability compared to ageand physical activity-matched controls, which is related to reduced aerobic capacity and muscle strength. CHF patients respond to resistance training with normal strength/functional adaptations. Our results support muscle weakness as a determinant of physical disability in CHF and show that interventions that increase muscle strength (resistance training) reduce physical disability.

\section{Keywords}

cachexia; sarcopenia; skeletal muscle; quality of life

Correspondence to: Michael J. Toth, Ph.D., Health Science Research Facility 126B, 149 Beaumont Ave, University of Vermont, Burlington, VT 05405, michael.toth@uvm.edu; Tele: (802)656-7989; Fax: (802)656-0747.

CONFLICTS OF INTEREST

The authors recognize no conflicts of interest. 


\section{INTRODUCTION}

Patients with chronic heart failure (CHF) have high rates of physical disability, based on self-reported difficulty in performing of activities of daily living (29). An impaired ability to perform simple everyday tasks reduces patients' quality of life, increases health care costs by increasing the need for supportive services and is an independent predictor of mortality $(10,11)$. Knowledge of the factors that determine physical function in CHF patients, therefore, has implications for improving quality of life and prognosis.

The ability to perform daily activities involves a complex interaction of physiological and psychological factors (6). In CHF patients, to our knowledge, no study has directly assessed disability by measuring performance in a range of common activities of daily living that encompass the physical skills required to function independently. The one exception is that many studies have evaluated 6 minute walk distance (28), a surrogate of aerobic fitness (13), as an index of functional capacity in daily activities. Arguably, this historical focus on indices of cardiorespiratory fitness is reasonable considering that the hallmark symptoms of CHF--dyspnea and fatigue --are emblematic of a reduction in aerobic capacity. Walking endurance, however, is only one aspect of physical function in daily activities. Moreover, performance in many daily activities has been shown to be poorly correlated to aerobic fitness in some cardiac disease populations (24), arguing that factors other than aerobic fitness likely contribute to physical disability. Compared to the number of studies that have measured indices of aerobic fitness, the relative paucity of studies examining muscle strength is surprising considering evidence for marked muscle weakness in CHF patients $(14,38)$ and the fact that many common daily activities (eg, lifting objects, rising from a seated position, climbing stairs, etc) are strongly dependent on muscle strength $(3,7,30)$. Despite the importance of muscle strength, its role in determining physical function in realworld activities of daily living in CHF patients has not been examined.

Numerous studies have demonstrated that aerobic training can improve exercise capacity, walking endurance and clinical status in CHF patients $(15,16,25)$. Despite these beneficial effects, aerobic training generally does not alter muscle strength (22) and, therefore, would not likely remediate aspects of physical disability associated with muscle weakness. Resistance-type exercise, in contrast, improves muscle strength, with little or no effect on aerobic fitness (32). Therefore, resistance exercise training may provide unique functional benefits to CHF patients. Additionally, it provides an experimental paradigm to evaluate the unique effects of improvements in muscle strength on physical function. Comparatively few studies, however, have examined the singular effects of resistance training in CHF patients, with most examining the combined effects of resistance and aerobic training (see for review $(8,34))$. Perhaps more importantly, aside from assessment of walking endurance (32), no study to our knowledge has explored the effects of resistance training on directly measured performance of activities of daily living to evaluate the real-world, functional benefits or resistance training in CHF patients.

The purpose of this study was two-fold: 1) to compare performance in activities of daily living in CHF patients and healthy controls of similar age and habitual physical activity level to evaluate the extent of physical disability and its relationship to aerobic capacity and muscle strength; and 2) to determine the effects of 18 weeks of resistance training on muscle strength and performance of activities of daily living. We hypothesized that CHF patients would be characterized by considerable physical disability and that this would be related to both diminished aerobic capacity and muscle weakness. Additionally, resistance exercise training effectively increases muscle strength and the ability to perform activities of daily living independent of alterations in aerobic fitness. To isolate the effects of CHF, we recruited patients to limit the confounding effects of age, muscle disuse and acute disease 
exacerbation. In light of these considerations, our results likely reflect the unique effects of CHF on physical disability, muscle strength and their response to training, rather then the effects of age, muscle disuse or acute illness.

\section{METHODS}

\section{Subjects}

Thirteen patients ( 9 men, 4 women) with CHF were recruited. Ten patients ( 7 men, 3 women; mean \pm SEM; age: $73.4 \pm 2.4$ yrs; height: $170.0 \pm 2.7 \mathrm{~cm}$; weight: $95.6 \pm 9.4 \mathrm{~kg}$ ) completed the trial and were included in the analyses. Of these ten patients, 7 were characterized by systolic failure with diminished ejection fraction (EF; $32 \pm 2 \%$, range $=28$ to $38 \%$ ), while 3 subjects had preserved systolic function $(\mathrm{EF}>40 ; 50 \pm 3 \%$, range $=44$ to 53\%). One patient was classified as New York Heart Association class I, 6 as class II and 3 as class III. The etiology of CHF was ischemic in 4 patients and idiopathic in 6. Patients were excluded if they had: 1) aute myocardial infarction or unstable angina within 3 months, 2) atrioventricular block greater than first degree without a functioning pacemaker, 3) severe hepatic or renal disease, 4) exercise-limiting peripheral vascular disease or orthopedic problems that limit their ability to perform exercise, 5) inflammatory arthritis or autoimmune disease or 6) an active neoplastic process or history of cancer within $5 \mathrm{yrs}$. To limit any effects of acute disease exacerbation/hospitalization on muscle performance and physical function, we included only patients who were clinically stable and had not been hospitalized for at least 6 months prior to study. Medications were maintained unchanged during the study and included: angiotensin-converting enzyme (ACE) inhibitors (100\%), beta-blockers (90\%), diuretics (50\%) and HMG CoA reductase inhibitors $(40 \%)$ and one female patient was receiving levothyroxine. The population included 3 patients with noninsulin dependent diabetes mellitus. Plasma creatine kinase levels were normal in all patients. All were weight-stable ( $\pm 2 \mathrm{~kg}$ during the prior 6 months), non-smokers and were not taking sex steroid replacement therapy.

All eleven ( 6 men, 5 women; age: $72.1 \pm 2.1$ yrs; height: $167.3 \pm 3.4$ cm; weight: $85.5 \pm 5.4$ $\mathrm{kg}$ ), healthy volunteers self-reported being sedentary to minimally-active ( $\mathcal{2}$ sessions of $230 \mathrm{~min}$ of exercise/week) and were not participating in any exercise or weight loss programs. This recruitment criteria was included to obtain a control group with habitual activity levels that match the reduced level of physical activity in the CHF population (36). Controls were non-smokers, weight-stable and not taking sex steroid replacement therapy and had no signs or symptoms of heart failure, coronary heart disease or diabetes. Left ventricular $\mathrm{EF}(>55 \%)$ was normal ( $62 \pm 4 \%$; range $=57$ to $70 \%)$, as were blood counts/ biochemical values. Five controls had hypertension. Three were treated with diuretics (27\%) and two with ACE inhibitors (18\%). All were normotensive at testing and showed no evidence of left ventricular hypertrophy or atrial enlargement by echocardiography. Three controls were on stable doses of HMG CoAs (27\%) and one female was on levothyroxine. Plasma creatine kinase levels were normal in all controls.

Written informed consent was obtained from all volunteers and the protocol was approved by the Committees on Human Research at the University of Vermont. Baseline, pre-training data from a sub-set of volunteers in this study have been published in reports of the effects of CHF on whole muscle and single fiber protein content and contractile performance and myosin-actin cross-bridge kinetics $(20,21,38)$. 


\section{Body composition}

Total and regional body composition was assessed by dual energy x-ray absorptiometry (GE Lunar, Madison, WI), as described previously (37). Body composition measurements were not performed on one CHF patient because he exceeded the weight limit of the machine.

\section{Echocardiography}

Left ventricular size and function was evaluated by echocardiography (Siemens Accuson Sequoia 512; Siemans Medical Solutions, Malvern, PA). Ventricular volumes and EF were determined by the biplane Simpsons' method (17).

\section{Peak oxygen consumption (peak $\mathrm{VO}_{2}$ )}

Respiratory gas analysis (SensorMedics Vmax 29c metabolic cart; Yorba Linda, CA) was performed during treadmill exercise until volitional exhaustion using the Naughton protocol (23).

\section{Directly measured performance in activities of daily living}

The Physical Functional Performance Test-10(PFP-10) is a validated battery of tests based on ordinary activities of daily life, performed at a self-paced, maximal effort (12). The PFP-10 tasks include: 1) transferring a weighted pot; 2) putting on and removing a jacket; 3 ) picking 4 scarves off the floor; 4) maximal overhead reach; $5 \& 6$ ) unloading and loading a washing machine and dryer; 7) getting down to a seated position on the floor and returning to a standing position; 8) grocery carry; 9) stair climbing; and 10) 6-minute walk. All tasks are performed with supervision and are quantified by time, distance and/or weight carried. Each task is scored 0-100, based on an empirically derived range of individual functional abilities (12). The test yields a total score (0-100) that is the average of the following 5 separate physical domains scores: 1) upper body strength; 2) lower body strength; 3 ) balance and coordination; 4) flexibility and 5) endurance. The PFP-10 has been validated over a broad range of functional levels to assess physical performance in healthy and diseased elderly (12), including older cardiac patients (3).

\section{Self-reported quality of life/physical function}

The Medical Outcomes Study Short-Form-36 (MOS SF-36) was used to assess "selfreported" physical function and quality of life, as described $(2,35)$, and has been validated in CHF patients (19).

\section{Exercise Protocol}

Approximately 1 week following baseline evaluations, volunteers entered an 18-week resistance training program $(3 \mathrm{X} / \mathrm{wk})$. The resistance exercise training program was designed to improve whole body skeletal muscle strength with the goal of determining whether improvements in muscle strength can improve functional performance in activities of daily living. The training intensity was set to $80 \%$ of $1 \mathrm{RM}$ commensurate with guidelines for improving muscle strength and inducing hypertrophy (5). The range of exercises, the progression of exercise volume and intensity and the length of the program were derived from our previous studies in healthy elderly and those with cardiac disease $(1,4,9)$, and are supported by data from others (32). Primary goals were to achieve significant strength gains and functional improvements. Additionally, we wanted the length of the program to be similar to most cardiac rehabilitation programs. Because this is the first study to assess the impact of resistance training on directly measured physical function in activities of daily living, our data have relevance for the clinical utility of resistance training for improving functional independence. 
Subjects were asked to not undertake any additional exercise during the study period. Compliance with the protocol was excellent (91\%) and was similar between CHF and control groups ( $47.6 \pm 2.1$ vs. $48.9 \pm 0.9$ sessions/patient, respectively, $\mathrm{P}=0.55)$. The original CHF cohort consisted of 13 patients. Three heart failure patients did not complete the training study: one because he became injured in a motor vehicle accident, another because of acute worsening of his heart failure and the last for personal reasons.

The resistance training intervention was individually designed based on 1-repetition maximum (1 RM; maximum weight an individual can lift once), as described in detail (4). At baseline, 1 RM was determined on each of 7 exercises, including: 1) leg extension; 2) leg press; 3) leg curls; 4) shoulder press; 5) bench press; 6) bicep curls; and 7) lateral pulldowns. A "composite 1 RM" was calculated by tallying the 1 RM for each of the 7 different exercises to provide an index of whole body muscle strength. Each session was supervised by an exercise physiologist or physical therapist. The progression of the program was gradual in both intensity and volume of exercise to orient the volunteers to the resistance training stimulus. The intensity of exercise began at 50\% 1RM for 1 set of 10 repetitions during the first week. On week 2, the intensity was increased to $60 \%$ for 2 sets of 8 repetitions. On week 3, the intensity was increased to $70 \%$ for 3 sets of 8 repetitions. By week 4, all volunteers were exercising at $80 \%$ of $1 \mathrm{RM}$ for 3 sets of 8 -repetitions. This ensured that the volunteers were exposed to the $80 \% 1 \mathrm{RM}$ stimulus for at least a 3 month period. 1RM was reassessed every 2 wks to account for improvements in strength. At the completion of the training program, all baseline evaluations were repeated, including 1 RM measurements. The only exception was echocardiography, which was repeated in CHF patients, but not controls.

\section{Statistic Analysis}

Unpaired $t$-tests were used to compare baseline values between groups. Baseline 1RM was compared between groups after statistical control for body weight using analysis of covariance. Pearson correlations were used to measure associations between variables. Repeated measures analysis of variance was used to assess group, training and group $\mathrm{X}$ training interaction effects. Paired t-tests were used to evaluate the effect of training on cardiac function in CHF patients. All statistical analyses were carried out using Stat View 5.01 (SAS Institute, Cary, NC).

\section{RESULTS}

\section{Baseline data}

At baseline, study groups were similar by age, sex distribution, body size and composition and physical activity; whereas, as expected, CHF patients had lower EF and peak $\mathrm{VO}_{2}$, and greater left ventricular end systolic volume (all $\mathrm{P}<0.001$; Table 1).

Muscle strength (1 RM) was similar between groups for all exercises, as was the composite 1 RM (Table 2). The lack of difference in muscle strength between groups was explained by variation among groups in body size. When composite $1 \mathrm{RM}$ was statistically controlled for body mass, whole body muscle strength was significantly reduced by $24 \%$ in CHF patients vs. controls ( $222.9 \pm 20.2$ vs. $293.5 \pm 19.3 \mathrm{~kg}$; $\mathrm{P}=0.023)$. Similarly lower $(25 \%)$ muscle strength was found in CHF patients when leg extension measurements were adjusted for body mass $(38.0 \pm 4.1$ vs. $50.6 \pm 3.9 \mathrm{~kg} ; \mathrm{P}=0.042)$, in keeping with our prior report examining knee extensor strength in a sub-set of this cohort using dynamometry (38). Additionally, after adjustment for body weight, $1 \mathrm{RM}$ for bench press $(-28.9 \% ; \mathrm{P}=0.031)$, shoulder press $(-34 \% ; \mathrm{P}=0.05)$ and leg press $(-15 \% ; \mathrm{P}=0.05)$ were lower in CHF patients; whereas, there was a strong trend towards a lower $1 \mathrm{RM}$ for leg curl $(-37 \% ; \mathrm{P}=0.061)$. 
Although no differences were found for $1 \mathrm{RM}$ values for lat pull down $(\mathrm{P}=0.125)$ or arm curl $(\mathrm{P}=0.172)$.

CHF patients had a lower $(\mathrm{P}<0.01)$ total PFP-10 score at baseline (Table 3), because of reduced $(\mathrm{P}<0.05$ to $\mathrm{P}<0.01)$ lower body strength, balance and endurance; whereas, upper body strength and flexibility were similar between groups. Additionally, baseline $6 \mathrm{~min}$ walk distance was reduced $(\mathrm{P}<0.001)$ in CHF patients. CHF patients self-reported lower physical and mental component summary scores as a result of lower $(\mathrm{P}<0.05$ to $\mathrm{P}<0.001)$ scores for: physical function, role-physical, bodily pain, general health, vitality, and social function ( $\mathrm{P}<0.05$ to $\mathrm{P}<0.001$; Table 4$)$.

Correlation analysis evaluated which physiological and psychological factors predict reduced physical function (Table 5). We examined both measured (i.e., PFP-10) and selfreported physical function (i.e., MOS SF-36 sub-domain) as indices of true physiological capacity for daily activities and self-perceived functional capacity, respectively, and also evaluated predictors of 6-minute walk performance, because it is a commonly used index of functional capacity in CHF populations. For directly measured functional capacity (ie, PFP-10 total score), peak $\mathrm{VO}_{2}(\mathrm{P}<0.001), 1 \mathrm{RM}$ composite $(\mathrm{P}<0.01)$ and MOS SF-36 mental component score $(\mathrm{P}<0.05)$ were significant correlates. For 6 min walk distance, the correlates were similar to PFP-10 total score, but also included EF $(\mathrm{P}<0.01)$. For selfreported physical function, peak $\mathrm{VO}_{2}$, measured physical functional capacity (ie, PFP-10 total score), MOS SF-36 Mental Component Score and ejection fraction (all, $\mathrm{P}<0.01$ ) were significant correlates, whereas 1RM composite was not.

\section{Resistance training data}

No training or group by training effects were observed for body weight, adiposity, appendicular skeletal muscle mass or peak $\mathrm{VO}_{2}$ (Table 1 and Figure 1). Resistance training increased $(\mathrm{P}<0.01$ training effect $)$ arm fat free mass and there was a trend toward an increase in fat free mass $(\mathrm{P}=0.057)$. CHF patients showed a greater increase in fat free mass with training when compared to controls $(\mathrm{P}=0.03$ group $\mathrm{X}$ training effect). For $\mathrm{CHF}$ subjects, there were no changes in LV volumes or EF with training.

Resistance training resulted in significant improvements in all 1 RM measures, including composite $1 \mathrm{RM}$ (all, $\mathrm{P}<0.001$ training effect; Table 2 and Figure 1) and these improvements did not differ between groups (ie, no group $\mathrm{X}$ training effect).

Total PFP-10 score improved with training in both groups $(\mathrm{P}<0.001$ training effect; Table 3 and Figure 1), as a result of improvements in upper and lower body strength and balance scores (P $₫ .02$ to 0.001 ); whereas, no improvements in upper body flexibility or endurance were noted. Training-induced increases in performance of activities of daily living were similar between groups (ie, no group $\mathrm{X}$ training effect). Training increased 6 minute walk distance in both groups similarly $(\mathrm{P}<0.05)$. Conversely, there was no training effect on MOS SF-36 scores (Table 4), although there were trends toward improvement in the physical function and mental health sub-domains $(\mathrm{P}=0.06$ and $\mathrm{P}=0.08$, respectively).

\section{DISCUSSION}

Our study is novel because it is the first to directly measure the extent of physical disability in common activities of daily living in CHF patients and its response to resistance exercise training. Our results demonstrate that CHF patients are markedly impaired in their physiological capacity to perform activities of daily living compared to controls of similar age and habitual physical activity level and that this impairment is associated with both reduced aerobic capacity and muscle weakness. Furthermore, resistance training is an 
effective intervention to enhance muscle strength and performance in activities of daily living. In light of our careful selection of volunteers to control for a variety of confounding disease-related factors, we are confident that group differences in physical function and muscle performance and their response to training reflect the unique effects of heart failure. Collectively, our findings support a role for muscle strength as a determinant of the physiological capacity to perform daily activities in CHF patients.

Although many studies have reported reduced walking endurance in CHF patients (ie, 6 min walk test (28)), this measure reflects but one facet of daily physical functioning. In the current study, we directly measured performance in a variety of real-world, daily activities that better reflect the range of physical attributes (ie, strength, endurance, balance and flexibility) required to function independently. Since CHF patients are profoundly inactive $(27,36)$, it could be argued that the extent of physical disability we observed is related to the secondary effects of chronic muscle disuse. This is unlikely since we recruited controls with similar habitual physical activity levels as CHF patients (Table 1) and tested patients distal to periods of acute muscle disuse (ie, hospitalization). Thus, we are confident that the observed functional differences are attributable to the effects of the CHF syndrome, rather than muscle disuse.

From a physiological standpoint, two of the most important factors determining physical function in the elderly are muscle strength and aerobic capacity (31). Although numerous studies have evaluated reduced aerobic capacity in CHF, the role of muscle weakness in physical disability, to our knowledge, has not been investigated. The focus on aerobic capacity is likely due to the fact that patients perceive their physical limitations through symptoms such as dyspnea and fatigue, which are emblematic of reduced aerobic fitness. In support of this notion, we found that self-reported functional capacity, as measured by the MOS SF-36, was correlated to aerobic capacity, but not to muscle strength. In contrast, our direct measurement of performance in activities of daily living (ie, PFP-10 score) was related to both aerobic capacity and muscle strength, substantiating a role for muscle strength as a determinant of physical function. These results highlight an often overlooked limitation of indices of self-reported physical function, which have formed the basis for nearly all of our current estimates of physical disability in the CHF population; namely, that they measure the patient's perception of their limitations, rather than their actual capacity to perform activities. Perhaps more importantly, although all of the metrics used in this study suggest a similar relative degree of physical disability $(\sim 30 \%$ lower functional capacity in CHF patients), the fact that their underlying determinants differ substantially (Table 5) demonstrates that each measure reflects a unique mix of the physiological and psychological determinants of physical disability. Our study provides the most rigorous assessment of the determinants of disability in CHF to date since we employed direct assessments of performance in activities of daily living as an index of physical disability. Our findings demonstrate that, as in populations of elderly without cardiac disease (31), muscle weakness conspires with diminished aerobic fitness to limit the capacity to perform daily activities in CHF.

Although it was not a goal of our study, the relative contribution of aerobic exercise intolerance and muscle weakness to physical disability deserves comment. Based solely on the strength of the statistical relationships, reduced aerobic capacity appears to be the more important determinant. However, peak $\mathrm{VO}_{2}$ measurements reflect more than just aerobic fitness level. For instance, muscle strength is a strong correlate of peak $\mathrm{VO}_{2}(14)$, which is likely explained by the fact that muscle force production is a determinant of power output during treadmill exercise (ie, contractile force $\mathrm{x}$ velocity $=$ muscle power output). In this sense, the correlation between peak $\mathrm{VO}_{2}$ and physical function reflects, in part, the effects of muscle strength. Additionally, because peak $\mathrm{VO}_{2}$ serves as a physiological index of disease 
severity in CHF patients $(18,39)$, its correlation to PFP-10 reflects, in part, the fact that HF patients were more functionally impaired. Both of these examples highlight the fact that peak $\mathrm{VO}_{2}$ has a high degree of colinearity with other determinants of physical disability, which complicates our ability to define its true contribution. There are statistical approaches to account for these problems of colinearity (33) and partial correlation analysis shows that control for the effects of $1 \mathrm{RM}$ composite score diminishes the relationship between peak VO2 and PFP-10 score (partial $r=0.695 ; \mathrm{P}<0.01$ ). However, caution is urged with drawing physiological conclusions from such statistical analysis that seeks to circumvent the fact that our study was not designed specifically to address this issue. Instead, we believe that delineation of the unique contributions of aerobic fitness and muscle strength to physical disability should be derived from studies that are carefully designed for this purpose.

Building on the last point, with respect to the unique effects of muscle strength on physical functional performance (ie, PFP-10 score), if the aforementioned correlation between muscle strength and function in daily activities reflects an underlying cause-effect relationship, we would predict that interventions which increase muscle strength, such as resistance training, would improve physical function. Consistent with this hypothesis, performance of activities of daily living was increased in CHF patients following 18-weeks of resistance training (Figure 1). In fact, improvements were similar to controls, suggesting that CHF does not impair neuromuscular adaptations to training in patients with mild to moderate disease. Perhaps more importantly, because peak $\mathrm{VO}_{2}$ was not altered with training, improvements in physical function are likely explained primarily by enhanced muscle strength, rather than altered aerobic capacity. We acknowledge that our resistance training program may have improved other factors that determine physical function, such as balance and flexibility. Thus, we cannot completely ascribe the improvements to improvements in muscle strength alone. Nonetheless, together with our correlation analyses, these findings strongly support a role for skeletal muscle strength as a determinant of performance in activities of daily living.

Participants with CHF had baseline total PFP-10 scores of 48.5, which is well below the score of 57 suggested by Cress et al. (12) as a threshold for physical disability. Although physical function improved with training in CHF patients to 53.9, it remained below this putative threshold. The inability of our training regimen to correct physical disability may relate to its short-term nature (4 months). The extent of muscle hypertrophy achieved during this period was minimal. Programs of longer duration that stimulate hypertrophy would certainly yield greater strength and functional benefits. Additionally, a singular intervention of resistance training may not be sufficient to correct disability in CHF patients because physical function is partially dependent on cardiorespiratory fitness (Table 5), which was not altered by our training program. Exercise regimens that combine both resistance and aerobic training may be needed to optimize functional improvements. Parenthetically, the fact that we observed no training-induced increase in physical activity level in CHF patients (via accelerometry) suggests that patients did not increase the amount of activity in their daily life despite their improved physiological capacity (ie, PFP-10 score). This result may be explained by psychological barriers to performing daily activities in CHF patients (26) and suggests the need for cognitive behavioral training to accompany exercise interventions so that patients can exploit their improved physiological capacity to reduce physical disability in their everyday life.

Limitations to the current study include the small number of subjects and a lack of participants randomized to a non-exercise control group. Importantly, our intent was not to conduct a randomized, controlled trial of the clinical efficacy of resistance training, as numerous studies have examined resistance training in $\mathrm{CHF}(8,34)$. Instead, we sought to examine the role of muscle strength as a determinant of physical disability in CHF patients 
and the effects of an exercise intervention that specifically enhances muscle strength to improve physical function. While we compromised statistical power with our approach, the rigorous selection criteria allowed us to experimentally remove a number of confounding factors from baseline disability levels and their responsiveness to training, a stronger experimental approach than post hoc statistical adjustment. Lending credibility to our results, the strength and functional (eg, 6 minute walk) improvements observed in our study were similar to those found in randomized, controlled trials utilizing similar intensity resistance training in CHF patients (32). Nonetheless, larger randomized, controlled trials are needed to assess the clinical utility of resistance training, alone and in combination with aerobic exercise, in reversing or delaying the onset of disability and improving clinical outcomes in CHF patients. Additionally, future studies should assess physical function and clinical outcomes following cessation of resistance exercise interventions to assess its longterm effects.

In summary, the ability to perform necessary activities of daily living is markedly impaired in CHF patients and is related to reduced aerobic capacity and muscle weakness. Resistance training is an effective intervention to improve strength and direct measures of physical function in CHF patients and these effects are independent of alterations in aerobic fitness. Collectively, these findings strongly support a role for muscle strength in determining the physiological capacity to perform activities of daily living. From a clinical perspective, our results suggest that interventions designed to lessen physical disability in CHF patients should consider improving muscle strength as one of their goals.

\section{Acknowledgments}

We thank all the volunteers who dedicated their valuable time to these studies. This study was funded by grants from the National Institutes of Health HL-077418 and RR-00109. The content is solely the responsibility of the authors and does not necessarily represent the official views of the National Heart Lung and Blood Institute or the National Institutes of Health, nor do the results of the study constitute endorsement by the American College of Sports Medicine.

\section{References}

1. Ades PA, Ballor DL, Ashikaga T, Utton JL, Nair KS. Weight training improves walking endurance in healthy elderly persons. Ann Intern Med. 1996; 124:568-72. [PubMed: 8597320]

2. Ades PA, Maloney A, Savage P, Carhart RL. Determinants of physical functioning in coronary patients. Response to cardiac rehabilitation. Arch Intern Med. 1999; 159:2357-60. [PubMed: 10547176]

3. Ades PA, Savage PD, Brochu M, Tischler MD, Lee NM, Poehlman ET. Resistance training increases total daily energy expenditure in disabled older women with coronary disease. J Appl Physiol. 2005; 98:1280-5. [PubMed: 15772059]

4. Ades PA, Savage PD, Cress ME, Brochu M, Lee NM, Poehlman ET. Resistance training on physical performance in disabled older female cardiac patients. Med Sci Sports Exerc. 2003; 35:1265-70. [PubMed: 12900677]

5. American College of Sports Medicine Position Stand. Progression models in resistance training for healthy adults. Med Sci Sports Exer. 2009; 41(3):687-708.10.1249/MSS.0b013e3181915670

6. Applegate WB, Blass JP, Williams TF. Instruments for the functional assessment of older patients. N Engl J Med. 1990; 322:1207-1214. [PubMed: 2183053]

7. Bean JF, Kiely DK, Herman S, et al. The relationship between leg power and physical performance in mobility limited elders. J Am Geriatr Soc. 2002; 50:461-7. [PubMed: 11943041]

8. Braith RW, Beck DT. Resistance exercise: training adaptations and developing a safe exercise prescription. Heart Fail Rev. 2008; 13:69-79. [PubMed: 17932746]

9. Brochu M, Savage P, Lee M, et al. Effects of resistance training on physical function in older disabled women with coronary heart disease. J Appl Physiol. 2002; 92:672-8. [PubMed: 11796680] 
10. Chaudhry SI, Wang Y, Gill TM, Krumholz HM. Geriatric conditions and subsequent mortality in older patients with heart failure. J Am Coll Cardiol. 2010; 55(4):309-16. [PubMed: 20117435]

11. Chiarantini D, Volpato S, Sioulis F, et al. Lower extremity performance measures predict longterm prognosis in older patients hospitalized for heart failure. J Cardiac Fail. 2010; 16(5):390-5.

12. Cress ME, Buchner DM, Questand KA, Esselman PC, deLateur BJ, Schwartz RS. Continuous scale-physical functional performance in a broad range of older adults: a validation study. Arch Phys Med Rehabil. 1996; 7:1243-50. [PubMed: 8976306]

13. Guyatt GH, Sullivan MJ, Thompson PJ, et al. The 6-minute walk: a new measure of exercise capacity in patients with chronic heart failure. Can Med Assoc J. 1985; 132:919-23. [PubMed: 3978515]

14. Harrington D, Anker S, Chua TP, et al. Skeletal muscle function and its relation to exercise tolerance in chronic heart failure. J Am Coll Cardiol. 1997; 30:1758-64. [PubMed: 9385904]

15. Haykowsky MJ, Liang Y, Pechter D, Jones LW, McAlister FA, Clark AM. A meta-analysis of the effect of exercise training on left ventricular remodeling in heart failure patients: the benefit depends on the type of training performed. J Am Coll Cardiol. 2007; 49(24):2329-36. [PubMed: 17572248]

16. Keteyian SJ, Pina IL, Hibner BA, Fleg JL. Clinical role of exercise training in the management of patients with chronic heart failure. J Cardiopulm Rehabil Prev. 2010; 30:67-76. [PubMed: 20216359]

17. Lang RM, Beierig M, Devereux RB, et al. Recommendations for chamber quantification: a report from the American Socieity of Echocardiography's Guidelines and Standards Committee and the Chamber Quantification Writing Group, Developed in conjunction with the European Association of echocardiography, a branch of the European Society of Cardiology. J Am Soc Echo. 2005; 18:1440-63.

18. Mancini DM, Eisen H, Kussmaul W, Mull R, Edmunds LH, Wilson JR. Value of peak exercise oxygen consumption for optimal timing of cardiac transplantation in ambulatory patients with heart failure. Circulation. 1991; 83:778-86. [PubMed: 1999029]

19. McHorney CA, Ware JE Jr, Lu JF, Sherbourne CD. The MOS 36-item Short-Form Health Survey (SF-36): III. Tests of data quality, scaling assumptions and reliability across diverse patient groups. Med Care. 1994; 32:40-66. [PubMed: 8277801]

20. Miller MS, VanBuren P, LeWinter MM, et al. Chronic heart failure decreases cross-bridge kinetics in single skeletal muscle fibers from humans. J Physiol. 2010; 588:4039-53. [PubMed: 20724360]

21. Miller MS, VanBuren P, LeWinter MM, et al. Mechanisms underlying skeletal muscle weakness in human heart failure: alterations in single fiber myosin protein content and function. Circ: Heart Fail. 2009; 2:700-6. [PubMed: 19919996]

22. Minotti JR, Johnson EC, Hudson TL, et al. Training-induced skeletal muscle adaptations are independent of systemic adaptations. J Appl Physiol. 1990; 68:289-94. [PubMed: 2312472]

23. Naughton J, Balke B, Nagle F. Refinements in method of evaluation and physical conditioning before and after myocardial infarction. Am J Cardiol. 1964; 14:837-43. [PubMed: 14232807]

24. Neill WA, Branch LG, DeJong G. Cardiac disability-the impact of coronary disease on patients' daily activities. Arch Intern Med. 1985; 145:1642-7. [PubMed: 4026494]

25. O'Connor CM, Whellan DJ, Lee KL, et al. Efficacy and safety of exercise training in patients with chronic heart failure: HF-ACTION randomized controlled trial. JAMA. 2009; 301(14):1439-50. [PubMed: 19351941]

26. Oka RK, Gortner SR, Stotts NA, Haskell WL. Predictors of physical activity in patients with chronic heart failure secondary to either ischemic or idiopathic dilated cardiomyopathy. Am J Cardiol. 1996; 77:159-63. [PubMed: 8546084]

27. Oka RK, Stotts NA, Dae MW, Haskell WL, Gortner SR. Daily physical activity levels in congestive heart failure. Am J Cardiol. 1993; 71:921-5. [PubMed: 8465782]

28. Olsson LG, Swedberg K, Clark AL, Witte KK, Cleland JGF. Six minute corridor walk test as an outcome measure for the assessment of treatment in randomized, blinded intervention trials of chronic heart failure: a systematic review. Eur Heart J. 2005; 26(8):778-93. [PubMed: 15774495] 
29. Pinsky JL, Jette AM, Branch LG, Kannel WB, Feinleib M. The Framingham Disability Study: relationship of various coronary heart disease manifestations to disability in older persons living in the community. Am J Pub Health. 1990; 80:1363-8. [PubMed: 2240306]

30. Ploutz-Snyder LL, Manini T, Ploutz-Snyder RJ, Wolf DA. Functionally relevant thresholds of quadriceps femoris strength. J Gerontol. 2002; 57:B144-B52.

31. Posner JD, McCully KK, Landsber LA, et al. Physical determinants of independence in mature women. Arch Phys Med Rehab. 1995; 76:373-80.

32. Pu CT, Johnson MT, Forman DE, et al. Randomized trial of progressive resistance training to counteract the myopathy of chronic heart failure. J Appl Physiol. 2001; 90:2341-50. [PubMed: 11356801]

33. Slinker BK, Glantz SA. Multiple regression for physiolgical data analysis: the problem of multicollinearity. Am J Physiol. 1985; 249:R1-R12. [PubMed: 4014489]

34. Spruit MA, Eterman R-MA, Hellwig VACV, Janssen PP, Wouters EFM, Uszko-Lencer NHMK. Effects of moderate-to-high intensity resistance training in patients with chronic heart failure. Heart. 2009; 95(17):1399-408. [PubMed: 19342376]

35. Stewart AL, Hays RD, Ware JE. The MOS short-form general health survey. Reliability and validity in a patient population. Med Care. 1988; 26:724-35. [PubMed: 3393032]

36. Toth MJ, Gottlieb SS, Goran MI, Fisher ML, Poehlman ET. Daily energy expenditure in freeliving heart failure patients. Am J Physiol. 1997; 272:E469-E75. [PubMed: 9124554]

37. Toth MJ, Matthews DE, Ades PA, et al. Skeletal muscle myofibrillar protein metabolism in heart failure: relationship to immune activation and functional capacity. Am J Physiol. 2005; 288:E685E92.

38. Toth MJ, Shaw AO, Miller MS, et al. Reduced knee extensor function in heart failure is not explained by inactivity. Int J Cardiol. 2010; 143:276-82. [PubMed: 19327849]

39. van den Broek SAJ, van Veldhuisen DJ, de Graeff PA, Landsman MLJ, Hillege H, Lie KI. Comparison between New York Heart Association classification and peak oxygen consumption in the assessment of functional status and prognosis in patients with mild to moderate chronic congestive heart failure secondary to either ischemic or idiopathic dilated cardiomyopathy. Am J Cardiol. 1992; 70:359-63. [PubMed: 1632403] 

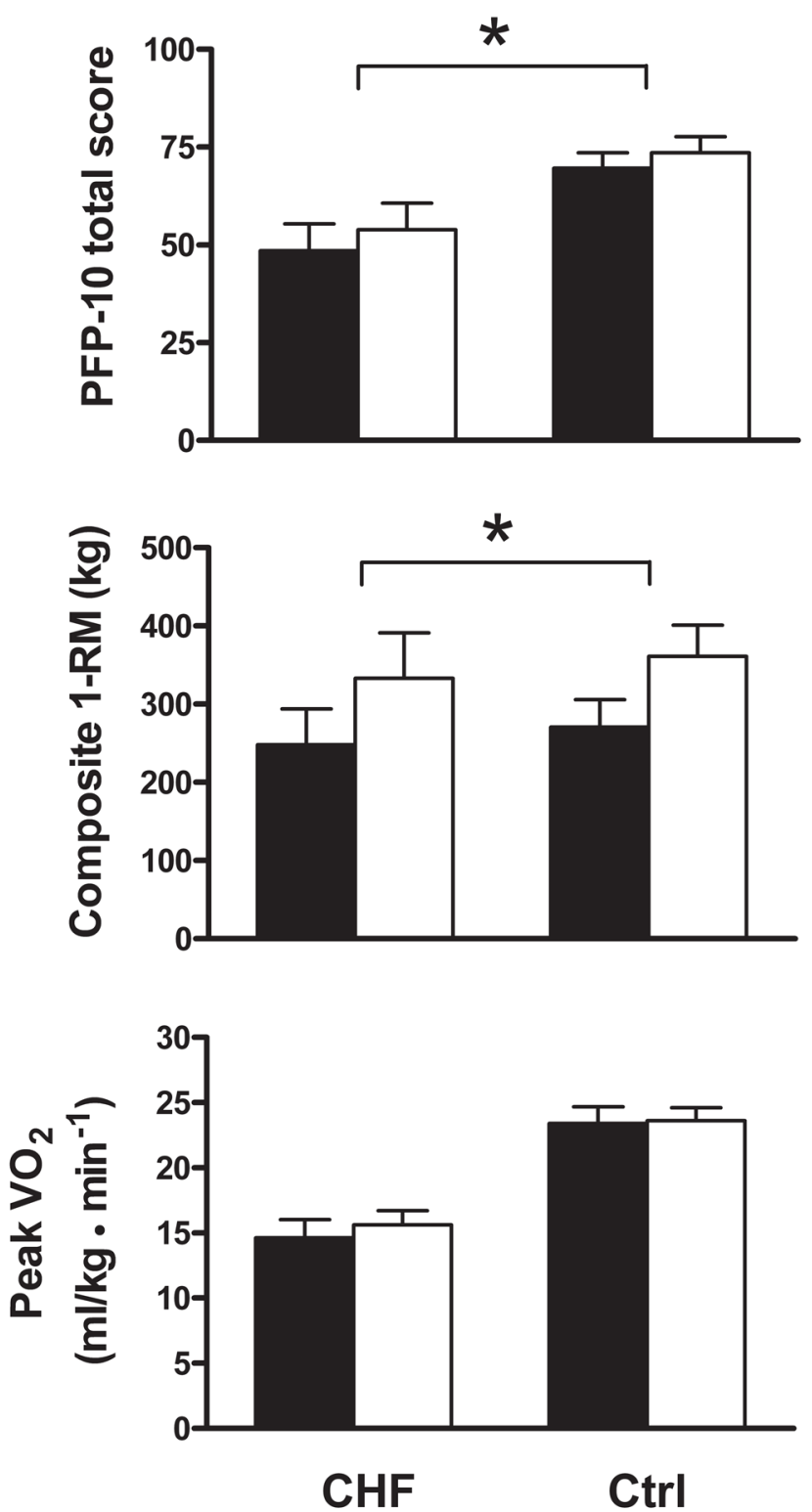

Figure 1.

Baseline (ie, pre; closed bars) and post-training (open bars) data for performance in activities of daily living (PFP-10 total score), composite $1 \mathrm{RM}$ and peak $\mathrm{VO}_{2}$ in chronic heart failure $(\mathrm{CHF})$ patients and controls $(\mathrm{Ctrl})$. Data were analyzed by $2 \times 2$ repeated measures analysis of variance to examine group, training and group by training interaction effects. Note that baseline PFP-10 and peak $\mathrm{VO}_{2}$ were lower in CHF patients compared to controls ( $\mathrm{P}<0.01$ and $\mathrm{P}<0.001$, respectively) and that composite $1 \mathrm{RM}$ was lower in $\mathrm{CHF}$ patients compared to controls $(\mathrm{P}<0.05)$ when statistically adjusted for body size. *, $\mathrm{P}<0.01$ for training effect. 


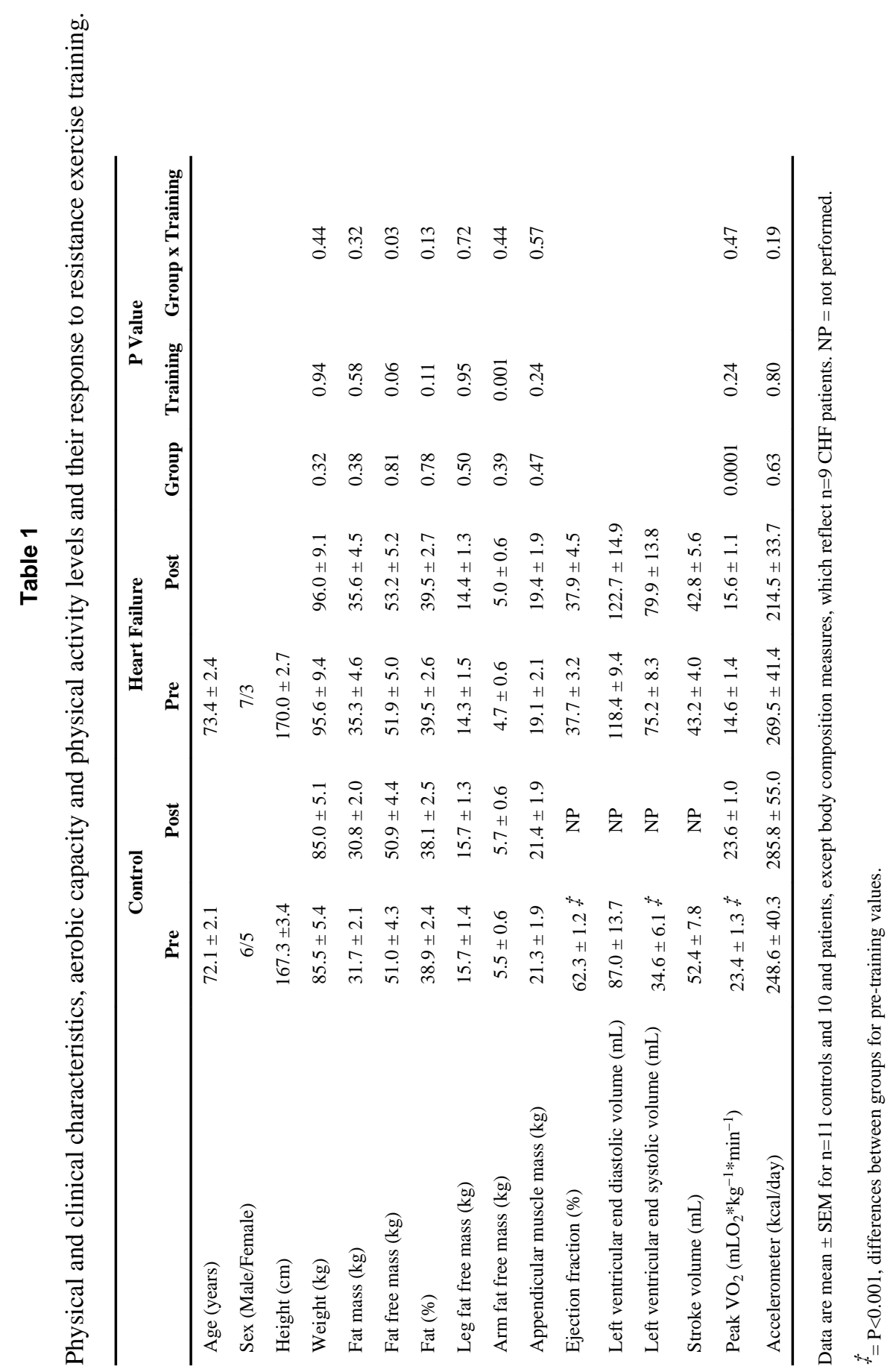




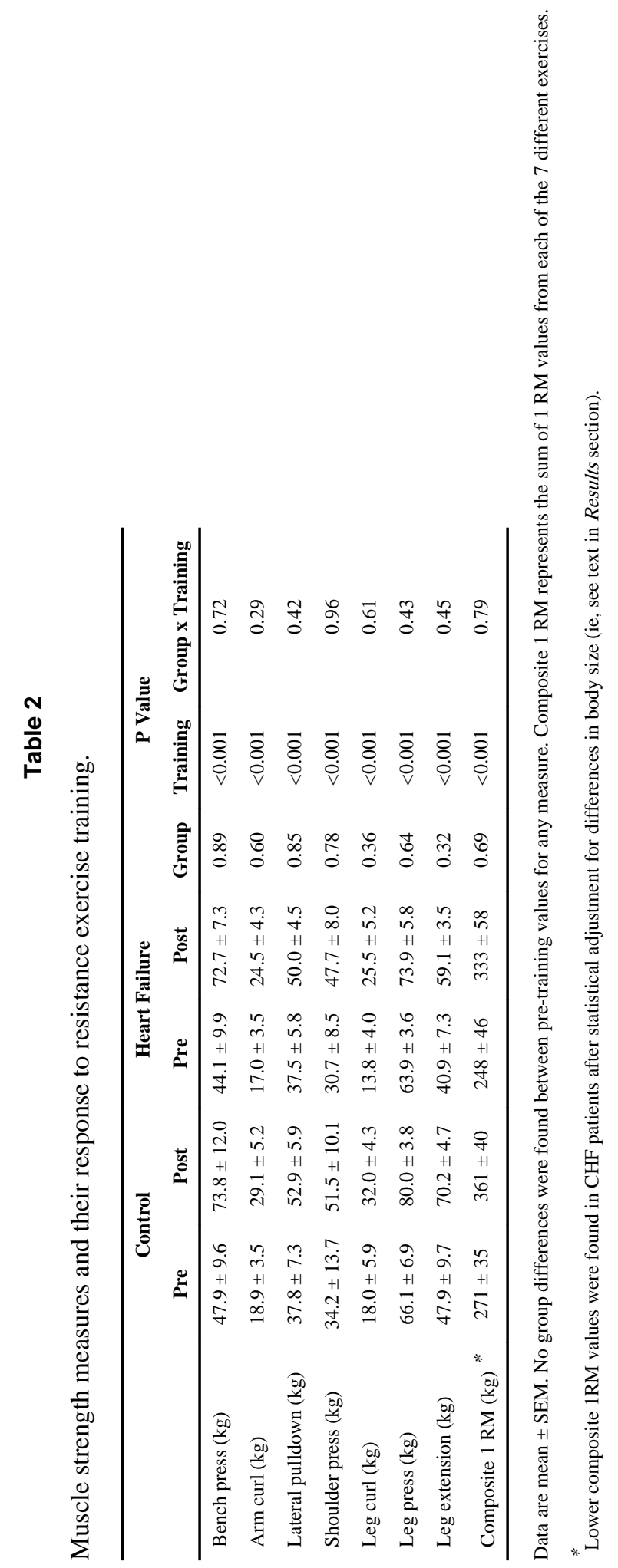

Med Sci Sports Exerc. Author manuscript; available in PMC 2012 August 02. 


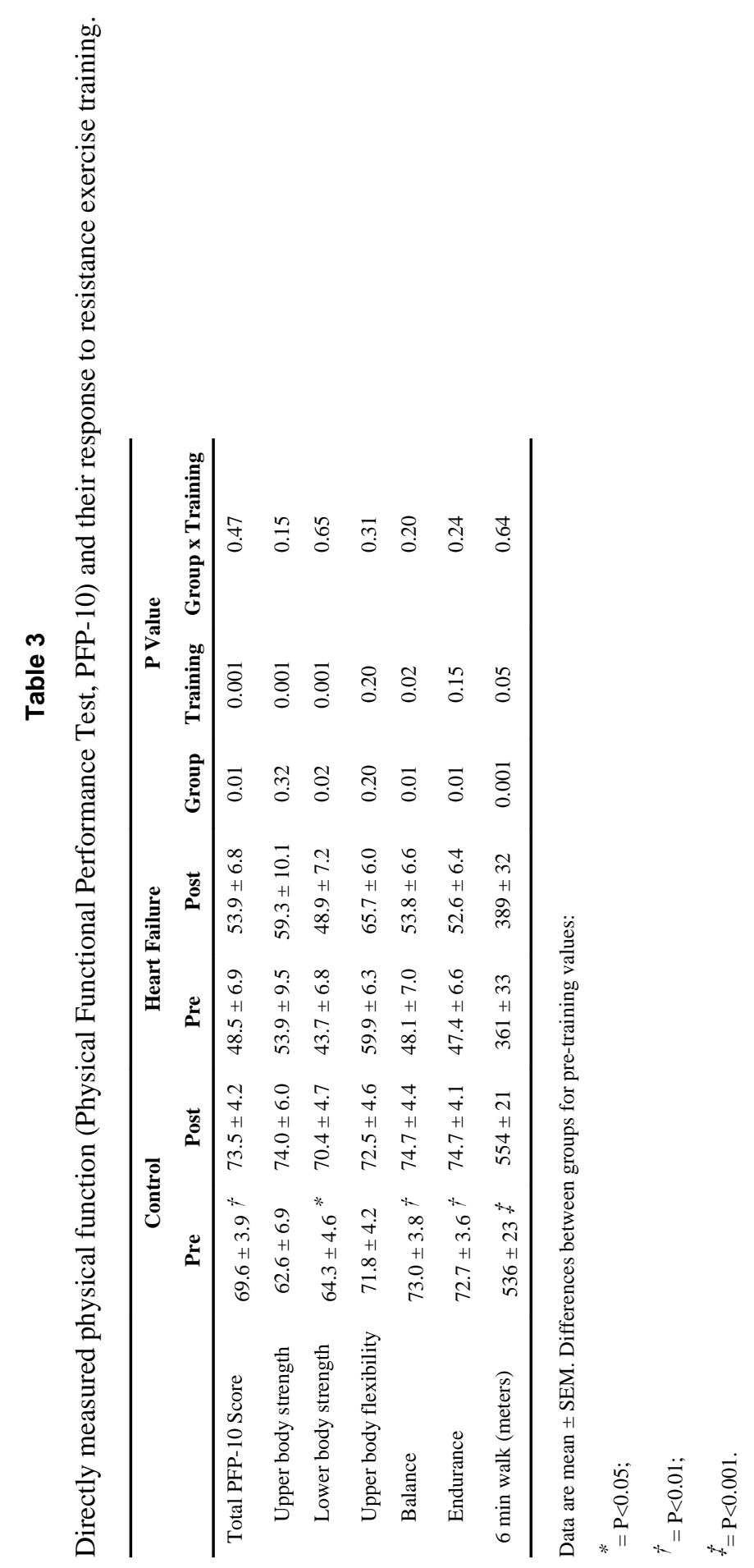

Med Sci Sports Exerc. Author manuscript; available in PMC 2012 August 02. 


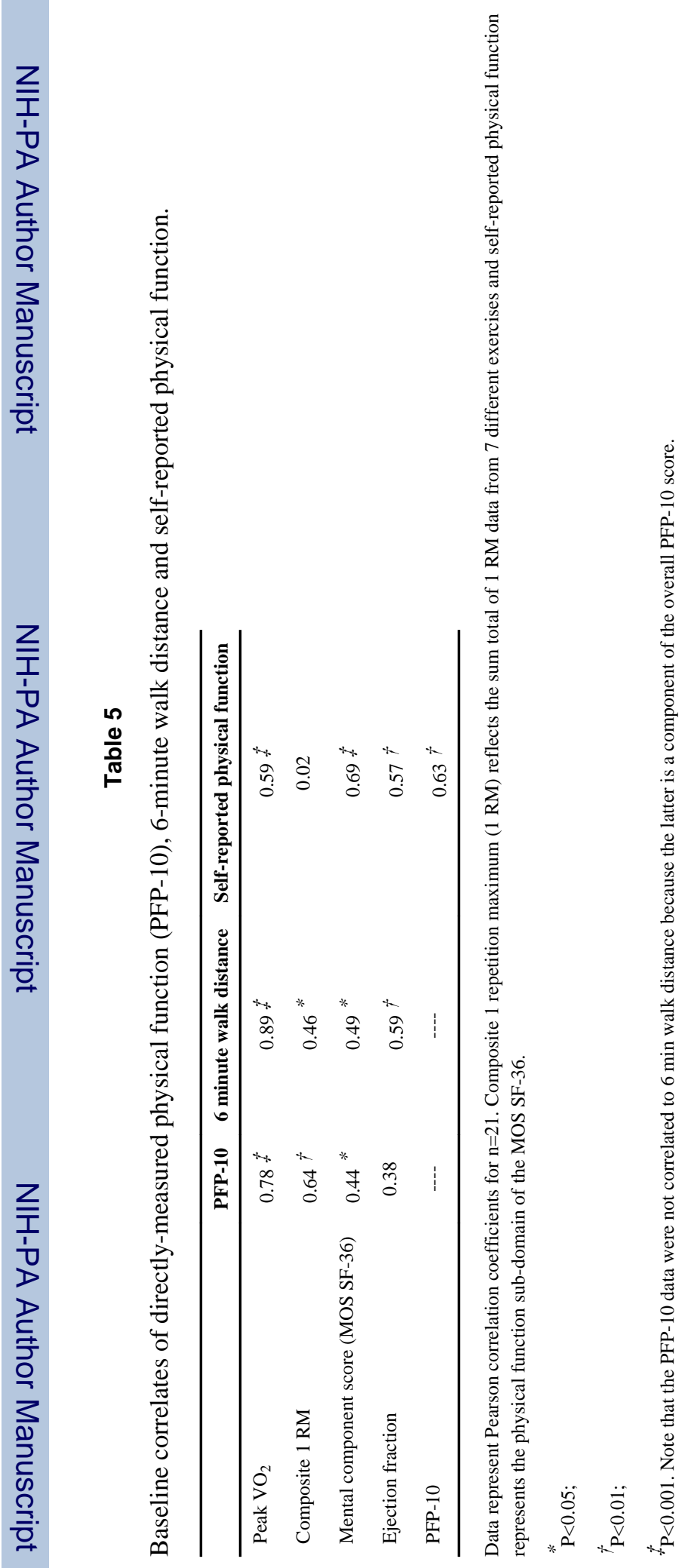

Med Sci Sports Exerc. Author manuscript; available in PMC 2012 August 02. 